\title{
Impact of Cone-Beam Computed Tomography with Automated Feeder Detection Software on the Survival Outcome of Patients with Hepatocellular Carcinoma During Chemoembolization
}

Kitttipitch Bannangkoon ( $\nabla$ drkittipitch@gmail.com )

Prince of Songkla University

Keerati Hongsakul

Prince of Songkla University

Teeravut Tubtawee

Prince of Songkla University

\section{Research Article}

Keywords: Cone-beam CT, Survival benefit, Local tumor recurrence, Hepatocellular carcinoma, Transarterial chemoembolization

Posted Date: October 22nd, 2021

DOl: https://doi.org/10.21203/rs.3.rs-953852/v1

License: (c) (1) This work is licensed under a Creative Commons Attribution 4.0 International License.

Read Full License 


\section{Abstract \\ Background}

Inoperable hepatocellular carcinoma (HCC) is treated by transarterial chemoembolization (TACE) using cone-beam computed tomography (CBCT) or digital subtraction angiography (DSA). We compared patient survival outcomes between CBCT-TACE with automated tumor-feeder detection (AFD) software and DSA-TACE alone in inoperable HCC patients.

\section{Methods}

We reviewed the data of 337 HCC patients treated by CBCT-TACE or DSA-TACE between January 2015 and December 2019. Treatment response, progression-free survival (PFS), overall survival (OS), and complications between the CBCT-TACE and DSA-TACE groups were compared. Univariate and multivariate logistic regression analyses examined the potential prognostic factors affecting survival after chemoembolization.

\section{Results}

Tumor response rates in complete response, partial response, and stable disease at 1 month were $67 \%$, $28 \%$, and $4 \%$ in the CBCT-TACE group and $22 \%, 48 \%$, and $9 \%$ in the DSA-TACE group, respectively. OS rates of patients in the CBCT-TACE vs DSA-TACE groups were $87 \%$ vs. $54 \%, 44 \%$ vs. $15 \%$, and $34 \%$ vs. $7 \%$ at 1,3 , and 5 years, respectively. The CBCT-TACE group had significantly improved PFS $(p<0.001)$ and OS $(p<0.001)$. Multivariate analysis showed that CBCT with AFD software was an independent factor associated with longer OS (hazard ratio, 0.38; $p<0.001$ ).

\section{Conclusions}

Compared with conventional DSA, combining selective TACE with CBCT and AFD software leads to better tumor response and prolongs OS in patients with inoperable HCC.

\section{Background}

Transarterial chemoembolization (TACE) is the standard treatment in patients with inoperable hepatocellular carcinoma (HCC), especially in Barcelona Clinic Liver Cancer (BCLC) stage B (intermediate stage) [1-3]. It is crucial to perform selective catheterization of all tumor-feeding arteries and precisely administer chemotherapeutic drugs and iodized oil to the tumor for effective TACE. However, inoperable stage HCC varies in terms of tumor size, number of tumor nodules, and complex multiple hepatic arterial branches that require multiple angiographic runs. Two-dimensional digital subtraction angiography (DSA) is routinely used for TACE procedures to identify the tumor-feeding branches and for tumor 
targeting. However, conventional DSA has some limitations in terms of identifying small tumor nodules and their feeding-arteries. Furthermore, DSA does not accurately assess accumulation of the iodized oil inside the tumors that might affect tumor recurrence and prognostic outcome $[4,5]$.

Nowadays, C-arm cone-beam computed tomography (CBCT) is a valuable tool for obtaining threedimensional images providing additional information including visualization of subtle tumor nodules, tiny hepatic feeding arteries, extrahepatic collateral arteries, and to monitor the embolized area during the TACE procedure [6-9]. Iwasawa et al. [10] reported that CBCT in addition to DSA during TACE can prolong survival in unresectable HCC patients.

Automated tumor-feeder detection (AFD) software is a new promising technology for the identification of intrahepatic and extrahepatic tumor-feeding arteries using CBCT data. The rates of identifying tumorfeeding arteries by AFD software were reported to be from $88-90 \%$ higher than using DSA guidance [7, $11,12]$. Accordingly, CBCT with AFD software has become an essential tool for performing TACE safely and effectively in the treatment of liver tumors. To our knowledge, the efficacy of intraprocedural CBCT with AFD software assisted TACE on survival outcome in unresectable HCC patients has not been identified in the literature. Therefore, for the first time, we retrospectively compared the survival outcome in patients who underwent CBCT using AFD software assisted TACE and patients who underwent TACE using DSA alone. We also evaluated the prognostic factors affecting the survival in HCC patients.

\section{Methods}

\section{Ethics statement}

This study complies with the standards of the Declaration of Helsinki and current ethical guidelines, and approval was obtained from the institutional ethics committee (REC No. 64-257-7-1). The requirement for informed consent for this study was waived by the Institutional Review Board, and all the data were analyzed anonymously.

\section{Patient population}

We followed the American Association for the Study of Liver Disease criteria for the diagnosis of HCC [13]. Hepatic lesions larger than $1 \mathrm{~cm}$ were assessed by multiphasic contrast-enhanced computed tomography (CT) or dynamic magnetic resonance imaging (MRI). If the imaging characteristics were the typical appearance of HCC, no further diagnostic procedure was attempted.

The data of 337 HCC patients treated with selective TACE between January 2015 and December 2019 at our hospital were collected for this study. Eligible patients for inclusion included: (1) patients aged $>18$ years, (2) HCC patients with tumor size $\leq 7 \mathrm{~cm}$ in diameter and the number of nodules $\leq 5$, (3) patients ineligible for surgical resection or transplantation, (4) patients treated with selective conventional TACE, and (5) patients who underwent imaging examination by either dynamic MRI or 4-phase contrastenhanced CT scan within one month after the initial procedure. The excluded patients were those with 
severe impaired hepatic function (Child-Pugh class C), extrahepatic metastasis, concomitant malignancy, severe arterioportal shunt, history of spontaneous tumor rupture, presence of vascular invasion, and cotreatment with any systemic or locoregional therapies during the TACE session.

Of the 337 HCC patients included in this study, 141 underwent TACE using DSA alone and 196 underwent TACE with CBCT (with or without AFD software) between January 2015 and December 2019. We assigned our patients to each technique on the basis of a discussion with interventional radiology staff. To minimize selection bias, patients treated by both techniques (i.e., first treated by TACE with DSA alone and subsequently underwent TACE by CBCT) were excluded from this study.

\section{Selective conventional TACE protocols}

All HCC patients underwent selective conventional TACE using an angiographic system (Allura Clarity FD20, Philips Healthcare, Eindhoven, the Netherlands) under the supervision of two interventional radiologists with more than 8 years of experience in body interventional radiology through the transfemoral route. Celiac and superior mesenteric arteries were selected at the beginning of the procedure using a 5 - $\mathrm{Fr}$ diagnostic catheter and a 0.035 -inch J-tip guidewire. We performed selective catheterization to the tumor feeding hepatic arteries or in extrahepatic collaterals as distal as possible in each tumor lesion using a 1.98-Fr to 2.4-Fr microcatheter. After the microcatheter was inserted into the target arterial feeder, we slowly administered a mixture of iodized oil (range, 2-16 ml) (Lipiodol, Guerbet) and doxorubicin hydrochloride (range, 5 to $50 \mathrm{mg}$ ) (Adriamycin, Pfizer) or mitomycin (range, 10-20 mg) (Vesimycin, Naprod Life Sciences) under real time monitoring with DSA. The amount of anticancer-in-oilemulsion (1:2 or 1:4 ratio) was adjusted by total tumor size and the number of nodules. Subsequently, the feeding artery was embolized using gelatin sponge particles. We completed the procedure when the tumor feeding branch was completely obstructed and tumor staining from DSA completely disappeared.

\section{Intraprocedural CBCT technique with AFD software}

The CBCT data[GKS1] were acquired[GKS2] using an Allura Clarity FD20 which enabled CBCT acquisition and volumetric image reconstruction. A total of 242 projection images ( 60 frames/second) with X-ray parameters of $121 \mathrm{kV}$ and $200-300 \mathrm{mAs}$ were obtained with the motorized C-arm covering a $220^{\circ}$ clockwise rotation of the flat panel detectors. The patients were instructed to be at end-expiratory apnea during the CBCT scanning. All CBCT images of $5 \mathrm{~mm}$ thickness were interpreted using a workstation (Philips Healthcare, Eindhoven, the Netherlands). We performed CBCT with AFD software assisted TACE in three steps (Fig. 1).

\section{Step 1: Identification of tumor nodules}

We placed the catheter at the proper or common hepatic artery and performed CBCT during hepatic arteriography (CBCTHA). A total of $24 \mathrm{ml}$ of non-diluted contrast media (Omnipaque $350 \mathrm{mgl} / \mathrm{ml}$, Bayer, Bangkok, Thailand) was injected via a 5-Fr diagnostic catheter at the rate of $2 \mathrm{ml} / \mathrm{second}$. The first phase of CBCTHA started after contrast material injection for 7 seconds and the second scan was achieved 30 
seconds after finishing the first scan. The patients held their breath twice during the gantry rotation. All hepatic lesions with corona enhancement, which represented venous drainage through hypervascular HCC nodules [14] on the second scan of CBCTHA were targeted for treatment with conventional TACE.

\section{Step 2: Detection of tumor feeding arteries}

The automated tumor-feeder software (EmboGuide, Philips Healthcare, Best, the Netherlands) used the data from the first scan of CBCTHA to identify the tumor feeders in about 2 minutes at the workstation. We used AFD software to detect segmental tumor feeders in case they were not clearly seen on conventional DSA images. After the feeder branches were identified, embolization was performed without obtaining further DSA images.

\section{Step 3: Evaluation of iodized oil accumulation:}

We performed plain CBCT without contrast media injection just after the selective TACE procedure to monitor the distribution of iodized oil. The goal of chemoembolization is to completely embolize the entire target tumor with or without an adequate safety margin. If incomplete tumor stains occurred, further chemoembolization was performed.

\section{Analysis of the prognostic factors for overall survival}

Univariate and multivariate analyses were used to define the significant independent factors affecting overall survival (OS). Fourteen clinical factors were analyzed: age, gender, alcohol drinking, hepatitis B or $C$ virus carrier, Child-Pugh class, albumin-bilirubin (ALBI) score, total bilirubin, albumin, international normalized ratio (INR), platelet count, serum alpha-fetoprotein (AFP) level, ascites, and BCLC staging. Also analyzed were four tumor factors: size of largest tumor, number of tumor nodules, lobar involvement, and the up-to-7 criteria plus two procedure-related factors: complete remission at 1 month and use of CBCT-assisted TACE.

\section{Outcome and treatment evaluation}

All eligible patients were followed up after selective TACE with a detailed clinical examination, blood chemistries, and an imaging study using 4-phase contrast-enhanced CT scan or dynamic MRI within one month after the initial procedure. If no definite evidence of residual or recurrent tumor presented, imaging investigation was performed subsequently at 3-month intervals. We used the modified Response Evaluation Criteria in Solid Tumors (mRECIST) to assess HCC after treatment [15]. Complete response (CR) was defined as disappearance of any intratumoral arterial enhancement in all target lesions. Partial response (PR) was defined as at least $30 \%$ decrease in the sum of the diameters of the target lesions. Progressive disease (PD) was defined as at least $20 \%$ increase in the sum of the diameters of the target lesions, and stable disease (SD) was defined as not sufficient to qualify for either PR or PD. The decision to repeat the TACE procedure was based on tumor response by MRECIST, BCLC-staging of the disease, and the patient's tolerance. 


\section{Complications}

TACE-related complications were classified as major or minor following the standard Society of Interventional Radiology (SIR) guidelines [16]. Major complications of conventional TACE were defined as admission to a hospital for therapy, an unplanned increase in the level of care, prolonged hospitalization, permanent adverse sequelae, or death after the procedures by SIR guidelines [16].

\section{Data and statistical analysis}

All data analyses were performed using R software (version 4.1.0). Numerical data are given descriptively using the central tendency (mean, median, and mode) and a measure of dispersion (standard deviation and range). We used chi-square or t-test to compare baseline characteristics between the HCC patients who underwent TACE with CBCT assistance and patients who received TACE with DSA. Local progression-free survival (PFS) was calculated from the date of selective TACE to the last date of local tumor progression or the date of patient death. Local tumor progression was diagnosed when an arterial enhancing lesion was depicted in or adjacent to the treated tumor on follow-up imaging. OS was calculated from the date of selective TACE to the date of patient death. Patient status at the end of the study (December 31, 2020) was defined as alive or dead using data from the Thailand civil registration database. The local PFS and OS rates for the two groups of HCC patients were compared using the Kaplan-Meier estimator. The probability of OS for the two groups was estimated using the Cox's proportional hazards regression model. The OS rates at 1, 3, and 5 years were calculated and compared for each group.

Baseline clinical characteristics, tumor appearances, and procedure-related factors affecting survival were initially assessed by univariate analysis. Subsequently, all prognostic factors having $p$ values $\leq 0.2$ from the univariate analysis were entered into the initial multivariate Cox's proportional hazards regression. The model was refined by sequentially removing non-significant variables. $P$ values $<0.05$ were considered statistically significant.

\section{Results}

\section{Patient characteristics}

A total of $337 \mathrm{HCC}$ patients were treated with selective TACE as the first treatment for HCC. One hundred and ninety-six patients were treated by TACE with CBCT assistance (CBCT-TACE group) and 141 patients were treated by TACE with DSA alone (DSA-TACE group). One hundred and fifty-one patients were categorized into BCLC-B stage and the rest were classified into BCLC stage 0 and stage A within the Milan criteria. Surgery or ablation was avoided in 186 patients in our study for these reasons: not eligible for surgery due to unfavorable remnant liver function, location of the tumor near a blood vessel or another 
organ, patient intention, inadequate visualization during ultrasonography, presence of ascites, advanced obesity, and severe abnormal coagulopathy.

Subsequently, baseline characteristics were compared between HCC patients in the CBCT-TACE group and patients in the DSA-TACE group (Table 1). There were no significant differences between the two groups in terms of age, gender, alcohol drinking, hepatitis B virus (HBV) positivity, hepatitis $C$ virus (HCV) positivity, Child-Pugh class, ALBI score, total bilirubin level, albumin level, INR level, platelet count, serum AFP level, presence of ascites, size of the largest tumor, tumor number, lobar involvement, up-to-7 criteria, and BCLC-staging. However, compared with HCC patients in the DSA-TACE group, the CBCT-TACE group had a considerably lower proportion of BCLC-B HCC patients $(p=0.130)$, and trends to have higher serum albumin $(p=0.064)$ and INR $(p=0.052)$ levels.

\section{TACE procedures}

A total of $628 \mathrm{HCC}$ nodules in 337 patients underwent selective TACE. The mean size of the largest tumor was $34.2 \pm 15.1 \mathrm{~mm}$ (range, $11-70 \mathrm{~mm}$ ) and the mean number of treated tumor nodules per TACE session was $1.9 \pm 1.2$ nodules (range, $1-5$ ). Most of the HCC patients had tumor nodules within the upto-7 criteria (79.2\%) and were characterized as unilobar involvement (84.0\%).

Procedural details and tumor response between HCC patients in the CBCT-TACE group and patients in the DSA-TACE group are summarized in Table 2. In the CBCT-TACE group, we performed selective TACE in 196 patients in 311 hepatic segmental areas. In the DSA-TACE group, we performed selective TACE in 141 patients in 213 hepatic segmental areas. The mean dose of iodized oil in one session of TACE was not significantly different between the two groups $(p=0.068)$. The mean dose of doxorubicin in one session of TACE was $21.6 \mathrm{mg}$ (range, 5-50 mg) in the CBCT-TACE group and $33.6 \mathrm{mg}$ (range, $5-50 \mathrm{mg}$ ) in the DSA-TACE group. The mean dose of doxorubicin in the DSA-TACE group was significantly higher than the CBCT-group $(p<0.001)$. The mean fluoroscopic time in one session of TACE in the CBCT-group was significantly longer than in the DSA-TACE group (16.3 vs. 12.4 minutes, $p<0.001$ ). However, the mean number of cine acquisitions for HCC patients in the CBCT group were significantly lower than the DSA group (4.6 vs. $5.1, p=0.017)$.

The mean number of CBCT acquisitions for the HCC patients in the CBCT-TACE group was 3.4 times (range, 1-11). In our study, CBCT was used to identify tumor nodules, detect tumor feeding arteries with AFD software, and evaluate iodized oil accumulation. First, of the 196 HCC patients in the CBCT-TACE group, the CBCT showed additional HCC nodules that were not evident on $\mathrm{CT}, \mathrm{MRI}$, or angiography in 34 patients (17.4\%). A total of 48 new HCC nodules were detected on CBCT images ( 1 new nodule in 23 patients, 2 new nodules in 8 patients, and 3 new nodules in 3 patients). The mean size of the newly detected HCC nodules was $10 \pm 3.1 \mathrm{~mm}$ (range, 5-21 mm). All newly found HCC nodules were subsequently treated with selective TACE. Second, we used the AFD software to detect all segmental tumor-feeding arteries in 139 of the 196 patients (70.9\%) when the feeding arteries were not clearly seen on conventional DSA images. The tumor feeding arteries were correctly detected by the AFD software in 133 of 139 patients $(95.7 \%)$. The reasons for failure to detect the feeding arteries in six patients were: 
motion artifacts (4 patients), hypovascular tumor nodule (1 patient), and very small caliber feeder vessel to the tumor (1 patient). Third, we monitored the distribution of the iodized oil immediately after the selective TACE procedure. If iodized oil deposition in the tumors was incomplete, further selective chemoembolization was performed.

Post-treatment assessments were evaluated by 4-phase contrast-enhanced CT scan or dynamic MRI 4 weeks after initial selective TACE. Tumor response rates by mRECIST in the CBCT-TACE group with CR, PR, SD, and PD were 67\% (131 patients), 28\% (55 patients), 4\% (7 patients), and 1\% (3 patients), respectively. In the DSA-TACE group, the CR, PR, SD, and PD response rates were $22 \%$ (31 patients), $48 \%$ (67 patients), 9\% (13 patients), and 21\% (30 patients), respectively. The response rates after selective TACE were significantly better in the CBCT-TACE group than in the DSA-TACE group $(p<0.001)$. The number of additional TACE procedures was not significantly different between the two groups $(p=$ $0.400)$.

\section{Complications}

All TACE-related complications were classified as minor. The most common procedure-related complication was post-embolization syndrome in 34 patients without requiring extended stay or readmission. Post-embolization syndrome was not significantly different between the CBCT-TACE and DSATACE groups $(p=0.920)$. Groin hematoma was found in eight patients without any treatment. Minimal iodized oil stains in the gallbladder wall due to non-target embolization without symptomatic cholecystitis was found in two patients in the DSA-TACE group without further treatment. Biloma occurred in one patient and was followed up without any treatment due to no symptoms. The remaining 221 patients were alive at the time of analysis.

\section{Progression-free survival}

The mean follow-up time for the HCC patients who underwent selective TACE was $20.0 \pm 15.3$ months (range, 0.4-71.1). The overall recurrence rate was about $96 \%$ (136/141 patients) in the DSA-TACE group and about 79\% (155/196 patients) in the CBCT-TACE group. The median tumor PFS periods for HCC patients in the DSA-TACE and CBCT-TACE groups were 33 days ( $95 \% \mathrm{Cl} 32-35$ days) and 467 days (95\% $\mathrm{Cl}$ 262-848 days), respectively. The median PFS rates for HCC patients in the CBCT-TACE group were significantly higher than in the DSA-TACE groups at 1 year ( $52 \%$ vs. $10 \%$ ), 3 years ( $38 \%$ vs. $6 \%$ ), and 5 years $(38 \%$ vs. $4 \%)$ by log-rank test $(p<0.001)$.

\section{Overall survival of patients in the CBCT-TACE and DSA-TACE groups}

The median OS time of the entire cohort was 23.9 months ( $95 \% \mathrm{Cl} 21.5-27.9$ months). The median OS times in the DSA-TACE and CBCT-TACE groups were 13.1 months (95\% $\mathrm{Cl} 11.4-16.8$ months) and 32.1 months (95\% Cl 29.5-46.4 months), respectively. The cumulative survival rates in the CBCT-TACE group were significantly higher than in the DSA-TACE group at 1 year ( $87 \%$ vs. $54 \%$ ), 3 years ( $44 \%$ vs. $15 \%$ ), and 
5 years $(34 \%$ vs. $7 \%)$ by log-rank test $(p<0.001)$ (Fig. 2$)$. The mortality rate was $91.5 \%$ (129/141 patients) in the DSA-TACE group and $46.9 \%$ (92/196 patients) in the CBCT-TACE group.

\section{Prognostic factors of overall survival}

Among the 20 prognostic factors affecting OS, univariate analysis revealed that Child-Pugh class $\mathrm{A}(p<$ $0.001)$, ALBI score 1 or $2(p<0.001)$, total bilirubin level $\leq 1.0 \mathrm{mg} / \mathrm{dl}(p<0.001)$, albumin $>3.5 \mathrm{~g} / \mathrm{dl}(p<$ $0.001)$, INR $\leq 1.2(p<0.001)$, serum AFP level $\leq 200 \mathrm{ng} / \mathrm{ml}(p=0.041)$, no presence of ascites $(p=$ $0.005)$, and BCLC staging 0 or $\mathrm{A}(p<0.001)$ were the significant clinical factors. Considering the tumor factors, size of largest tumor $\leq 3 \mathrm{~cm}$ in diameter and single HCC nodule were significant tumor factors on univariate analysis ( $p=0.012$ and $p=0.011)$. In addition, use of CBCT assisted TACE and presence of CR at 1 month after TACE were significant procedure-related factors $(p<0.001$ and $p<0.001)$ (Table 3$)$. Multivariate analysis for the potential prognostic factors affecting OS showed that ALBI score 1 or 2 (hazard ratio [HR]: $0.48 ; p<0.001$ ), serum AFP level $\leq 200 \mathrm{ng} / \mathrm{ml}$ (HR: $0.63 ; p=0.003$ ), presence of CR at 1 month after TACE (HR: $0.55 ; p<0.001)$, and use of CBCT assisted TACE (HR: $0.38 ; p<0.001)$ were the only four independently significant prognostic factors associated with longer OS (Table 3 ).

\section{Discussion}

In our study, the additional information provided by $\mathrm{CBCT}$ resulted in a change in treatments, including visualization of angiographically occult HCC nodules in 34 patients (17.4\%) in the CBCT-TACE group, that were not demonstrated on CT, MRI, or angiography. All 48 small HCC nodules (mean diameter, $10.0 \pm 3.1$ $\mathrm{mm}$ ) that could not be detected on other images could be depicted by dual-phase CBCTHA. These findings suggested that the sensitivity of HCC detection increases using CBCT assistance, as reported in previous studies $[8,17,18]$. A meta-analysis study $[8]$ showed that the sensitivity of CBCT to detect a HCC tumor was $90 \%$, whereas the sensitivity of DSA for tumor detection was only $67 \%$. Additionally, nontumorous lesions mimicking HCCs are often seen on CBCT images that result in reduced specificity of tumor detection [14]. As a result, we used the corona enhancement on second phase CBCTHA images to distinguish between HCC nodules and hypervascular pseudolesions. Corona enhancement represents venous drainage through hypervascular HCC and is detected in $89 \%$ of HCC nodules [19]. On the contrary, pseudolesions, such as arterioportal shunts, do not demonstrate corona enhancement [18]. Therefore, our technique in dual-phase CBCTHA depiction of corona enhancement can improve the diagnosis accuracy of HCC nodules. Subsequently, all HCC lesions with corona enhancement on the second scan of CBCTHA were targeted for selective TACE.

The volumetric information from CBCT provides a rotational three-dimensional map of the hepatic arterial anatomy and detection of tumor-feeding branches for selective TACE. Since the development of AFD software to identify tumor feeding vessels using CBCT data during hepatic arteriography, we now routinely use TACE guidance software when tumor-feeding arteries are not clearly seen on conventional DSA images. AFD assessment was generated in about 2 minutes at the workstation. Based on our study, tumor feeders were correctly detected by the AFD software in $95.7 \%$ (133/139 patients), which was 
similar to previous studies $[6,20,21]$ that reported the detectability rate of the vessel tracking software was about $81-93 \%$. When a tumor-feeding branch was identified by AFD software, we performed selective TACE of that branch without performing additional DSA runs. These results reduced the number of cine acquisitions in the CBCT group compared to the DSA group (4.6 vs. $5.1 ; p=0.017$ ). A previous study [22] demonstrated that the feeder-vessel detection software also reduced the overall procedural time in TACE. However, mean fluoroscopic time in the CBCT-TACE group was significantly longer than in the DSA-TACE group (16.3 vs. 12.4 minutes, $p<0.001$ ). This occurred probably because the patients in the CBCT-TACE group had small and subtle tumor-feeding branches that required more fluoroscopic time for selective catheterization. Some limitations of CBCT images are due to motion artifacts caused by inadequate breath-holding. We found that motion artifacts causing failure to detect feeding arteries using AFD software was $2.9 \%$ in the CBCT-TACE group, which was similar to the $3 \%$ of patients in a previous report [23]. We can reduce these motion errors by training the patients to hold their breath before the procedures and performing the dual-phase CBCTHA in a propeller-fashion (from the head-end of the table) that requires a scan time of 5 seconds. However, the rotation time in a roll-fashion (from the side of the table) is usually longer than 8 seconds, which is more difficult for the patient to achieve a single breath hold.

The goal of selective TACE is complete embolization of the target HCC nodule with an adequate safety margin that leads to a good therapeutic outcome and lower rate of tumor recurrence [9]. CBCT during selective TACE can verify the accumulation of iodized oil and guide the operator on the endpoint of chemoembolization. If, immediately after selective TACE, the CBCT shows a non-enhancing portion within the hypervascular HCC nodule, it indicates one of three possibilities: (1) incomplete embolization of the feeding artery, (2) some small feeding arteries were missed, or (3) the presence of an extrahepatic collateral artery supplying the tumor. Although the signal-to-noise ratio of a CBCT image is lower compared with multidetector $\mathrm{CT}$, the $\mathrm{CBCT}$ is nearly comparable to the multidetector CT for identifying incomplete iodized oil accumulation after TACE [5].

In our study, the objective response rate (OR) by mRECIST in the CBCT-TACE group was about 95\%, compared to the 70\% OR in the DSA-TACE group. The tumor response rate after selective TACE was significantly better in the CBCT-TACE group than in the DSA-TACE group $(p<0.001)$, even though the mean dose of chemotherapeutic drugs was significantly lower in the CBCT-TACE group. These findings suggested that modification of the procedure with TACE guidance software can decrease local tumor recurrence and improve the tumor response, which were similar to a previous report [24] while reducing the amount of chemoembolic agents. Although post-embolization syndrome was not significantly different between the CBCT-TACE and DSA-TACE groups in our study, iodized oil stains in the gallbladder were found only in two patients in the DSA-TACE group. It was reported that non-target embolization after TACE through the cystic artery can cause serious complications, such as gallbladder infarct, that require surgery [25]. The CBCT with AFD software can also provide information on the vascular anatomy including non-hepatic arteries originating from the hepatic artery (i.e., cystic artery, accessory left gastric artery, right gastric, and falciform artery), to prevent non-target embolization. 
In 2016, Lencioni et al. [3] reported in a systematic review that in 10,108 patients treated with lipiodol TACE the OS rates at 1,3 , and 5 years were $70 \%, 40 \%$, and $32 \%$, respectively, and the median OS time was 19.4 months ( $95 \% \mathrm{Cl} 16.2-22.6)$. In our study, the overall survival rates of the patients in the CBCT-TACE group with AFD software guidance at 1,3 , and 5 years were $87 \%, 44 \%$, and $34 \%$, respectively, and the median OS time in the CBCT-TACE group was 32.1 months ( $95 \% \mathrm{Cl} 29.5-46.4$ months). Furthermore, our study also determined that use of CBCT assisted TACE is an independently significant prognostic factor associated with longer OS (HR: $0.38 ; p<0.001$ ), which was similar to a previous study by Iwazawa et al. [10]. However, there are some differences in terms of methodology. In a previous study [10], the tumorfeeding branch that supplied the target tumor was clarified manually from CBCT images and confirmed by testing with contrast injection to determine whether the entire tumor was totally enhanced. In contrast, we routinely used AFD software to detect segmental tumor-feeding arteries when they were not clearly seen on conventional DSA images. When the feeder branches were labeled from the TACE guidance software, the branches were embolized without performing additional DSA images. In the Iwazawa study [10], the median OS time in the CBCT-TACE group without AFD software was 27.2 months ( $95 \% \mathrm{Cl} 0.7-$ 49.3 months), which was shorter compared to the CBCT-TACE group with AFD software guidance in our study (32.1 months, 95\% Cl 29.5-46.4 months). Therefore, CBCT with TACE guidance software contributed to longer patient survival compared to HCC patients who underwent TACE with DSA guidance and was possibly higher than the patients treated with CBCT aided TACE but without AFD software assistance.

In the present study, ALBI score, serum AFP $\leq 200 \mathrm{ng} / \mathrm{ml}$ and presence of CR at 1 month after TACE were independently significant prognostic factors associated with longer OS. The ALBI score plays an important role in the assessment of hepatic reserve function after TACE as well as the importance of judging TACE failure in clinical practice. These findings were similar to a few reports where the ALBI score was associated with OS in HCC patients treated with TACE [26, 27]. AFP plays crucial functions in both immune system inhibition and promoting cancer cell growth. Previous studies [28, 29] found that high AFP serum levels were associated with a poorer differentiation grade of HCC and a higher rate of tumor recurrence. In principle, the baseline tumor burdens (tumor size and number), tumor biology (sensitivity to chemotherapeutic drugs), and procedure-related factors (vascular accessibility for intervention) play important roles in establishing overall success rates of TACE procedures [30, 31]. In particular, smaller tumor size and limited tumor number are significantly associated with success in achieving CR immediately after the first TACE session and related with favorable outcomes [32].

Our study has some limitations. First, this study was retrospective in nature conducted at a single center. Second, we did not evaluate the types of microcatheters, skill of the TACE operators or level of selective catheterization that may influence the interpretation of the survival outcome. Third, histological confirmation of HCC was not obtained. All study lesions were diagnosed as HCC based on imaging findings and elevated serum levels of tumor markers. Finally, our sample size was relatively small, which limited the number of patients available for a subgroup analysis. 
In conclusion, the CBCT technology with AFD software can provide additional information during selective TACE including identification of HCC nodules, detection of tumor feeding arteries as a 3D navigation tool, and evaluation of iodized oil accumulation, which affects the treatment response and prolongs survival in patients with inoperable HCC. The combination of selective TACE with the capabilities of CBCT and TACE guidance software in daily clinical practice may improve the efficacy and prognostic outcome of HCC patients.

\section{Declarations}

\section{Acknowledgements}

The authors would like to express our deep and sincere gratitude to Assoc. Prof. Teerha Piratvisuth, from the NKC Institute of Gastroenterology and Hepatology, Faculty of Medicine, Prince of Songkla University for giving me invaluable suggestions from the beginning to the end of this study. We would like to thank Mrs. Jirawan Jayuphan, from Epidemiology Unit, Faculty of Medicine, Prince of Songkla University for statistics work.

\section{Authors' contributions}

Study concept and design: K.B, K.H., T.T.

Data acquisition: K.B, T.T.

Data analysis and interpretation: K.B, K.H.

Drafting of the manuscript: K.B, K.H.

Critical revision of the manuscript for important intellectual content: K.B, K.H.

Statistical analysis: K.B, K.H., T.T.

All authors approved the final draft of the manuscript submitted to the journal.

\section{Funding}

This research received no specific grant from any funding agency in the public, commercial, or not-forprofit sectors.

\section{Availability of data and material}

All analyzed data are included in this published article. The original data are available upon reasonable request to the corresponding author.

\section{Ethics approval and consent to participate}


This study complies with the standards of the Declaration of Helsinki and current ethical guidelines, and approval was obtained from the institutional ethics committee (REC No. 64-257-7-1). The requirement for informed consent for this study was waived by the Institutional Review Board, and all the data were analyzed anonymously.

\section{Consent for publication}

Not applicable.

\section{Competing interests}

The authors declare no competing interests.

\section{References}

1. Lo CM, Ngan H, Tso WK, et al. Randomized controlled trial of transarterial lipiodol chemoembolization for unresectable hepatocellular carcinoma. Hepatology. 2002;35:1164-71.

2. Llovet JM, Real MI, Montaña X, et al. Barcelona Liver Cancer Group. Arterial embolisation or chemoembolisation versus symptomatic treatment in patients with unresectable hepatocellular carcinoma: a randomised controlled trial. Lancet. 2002;359:1734-9.

3. Lencioni R, de Baere T, Soulen MC, Rilling WS, Geschwind JF. Lipiodol transarterial chemoembolization for hepatocellular carcinoma: A systematic review of efficacy and safety data. Hepatology. 2016;64:106-16.

4. Iwazawa J, Ohue S, Mitani T, et al. Identifying feeding arteries during TACE of hepatic tumors: comparison of C-arm CT and digital subtraction angiography. AJR Am J Roentgenol. 2009;192:105763.

5. Iwazawa J, Ohue S, Kitayama T, Sassa S, Mitani T. C-arm CT for assessing initial failure of iodized oil accumulation in chemoembolization of hepatocellular carcinoma. AJR Am J Roentgenol. 2011;197:W337-42.

6. Deschamps F, Solomon SB, Thornton RH, et al. Computed analysis of three-dimensional cone-beam computed tomography angiography for determination of tumor-feeding vessels during chemoembolization of liver tumor: a pilot study. Cardiovasc Intervent Radiol. 2010;33:1235-42.

7. Miyayama S, Yamashiro M, Hashimoto M, et al. Identification of small hepatocellular carcinoma and tumor-feeding branches with cone-beam CT guidance technology during transcatheter arterial chemoembolization. J Vasc Interv Radiol. 2013;24:501-8.

8. Pung L, Ahmad M, Mueller K, et al. The Role of Cone-Beam CT in Transcatheter Arterial Chemoembolization for Hepatocellular Carcinoma: A Systematic Review and Meta-analysis. J Vasc Interv Radiol. 2017;28:334-341.

9. Bannangkoon K, Hongsakul K, Tubtawee T, Piratvisuth T. Safety margin of embolized area can reduce local recurrence of hepatocellular carcinoma after superselective transarterial 
chemoembolization. Clin Mol Hepatol. 2019;25:74-85.

10. Iwazawa J, Ohue S, Hashimoto N, Muramoto O, Mitani T. Survival after C-arm CT-assisted chemoembolization of unresectable hepatocellular carcinoma. Eur J Radiol. 2012;81:3985-92.

11. Miyayama S. Ultraselective conventional transarterial chemoembolization: When and how? Clin Mol Hepatol. 2019;25:344-353.

12. Cui Z, Shukla PA, Habibollahi P, Park HS, Fischman A, Kolber MK. A systematic review of automated feeder detection software for locoregional treatment of hepatic tumors. Diagn Interv Imaging. 2020;101:439-449.

13. Bruix J, Sherman M; American Association for the Study of Liver Diseases. Management of hepatocellular carcinoma: an update. Hepatology. 2011;53:1020-2.

14. Kim HC. Role of C-arm cone-beam CT in chemoembolization for hepatocellular carcinoma. Korean J Radiol. 2015;16:114-24.

15. Llovet JM, Lencioni R. mRECIST for HCC: Performance and novel refinements. J Hepatol. 2020;72:288-306.

16. Brown DB, Cardella JF, Sacks D, et al. Quality improvement guidelines for transhepatic arterial chemoembolization, embolization, and chemotherapeutic infusion for hepatic malignancy. J Vasc Interv Radiol. 2009;20:S219-S226, S226.e1-10.

17. Iwazawa J, Ohue S, Hashimoto N, Abe H, Hamuro M, Mitani T. Detection of hepatocellular carcinoma: comparison of angiographic C-arm CT and MDCT. AJR Am J Roentgenol. 2010;195:882-7.

18. Miyayama S, Yamashiro M, Hattori Y, et al. Efficacy of cone-beam computed tomography during transcatheter arterial chemoembolization for hepatocellular carcinoma. Jpn J Radiol. 2011;29:371-7.

19. Ueda K, Matsui O, Kawamori Y, et al. Differentiation of hypervascular hepatic pseudolesions from hepatocellular carcinoma: value of single-level dynamic CT during hepatic arteriography. J Comput Assist Tomogr. 1998;22:703-8.

20. Iwazawa J, Ohue S, Hashimoto N, Muramoto O, Mitani T. Clinical utility and limitations of tumorfeeder detection software for liver cancer embolization. Eur J Radiol. 2013;82:1665-71.

21. Miyayama S, Yamashiro M, Ikuno M, Okumura K, Yoshida M. Ultraselective transcatheter arterial chemoembolization for small hepatocellular carcinoma guided by automated tumor-feeders detection software: technical success and short-term tumor response. Abdom Imaging. 2014;39:64556.

22. Imazawa J, Ohue S, Hashimoto N, Mitani T. Comparison of the Number of Image Acquisitions and Procedural Time Required for Transarterial Chemoembolization of Hepatocellular Carcinoma with and without Tumor-Feeder Detection Software. Radiol Res Pract. 2013;29:580839.

23. Lee IJ, Chung JW, Yin YH, et al. Cone-beam CT hepatic arteriography in chemoembolization for hepatocellular carcinoma: angiographic image quality and its determining factors. J Vasc Interv Radiol. 2014;25:1369-79; quiz 1379-.e1. 
24. Miyayama S, Yamashiro M, Hashimoto M, et al. Comparison of local control in transcatheter arterial chemoembolization of hepatocellular carcinoma $\leq 6 \mathrm{~cm}$ with or without intraprocedural monitoring of the embolized area using cone-beam computed tomography. Cardiovasc Intervent Radiol. 2014;37:388-95.

25. Collins J, Salem R. Hepatic radioembolization complicated by gastrointestinal ulceration. Semin Intervent Radiol. 2011;28:240-5.

26. Khalid MA, Achakzai IK, Hanif FM, Ahmed S, Majid Z, Luck NH. To determine the prognostic value of the albumin-bilirubin grade (ALBI) in patients underwent transarterial chemoembolization for unresectable hepatocellular carcinoma. Gastroenterol Hepatol Bed Bench. 2019 Spring;12(:110-115.

27. Izumoto H, Hiraoka A, Ishimaru Y, et al. Validation of Newly Proposed Time to Transarterial Chemoembolization Progression in Intermediate-Stage Hepatocellular Carcinoma Cases. Oncology. 2017;93 Suppl 1:120-126

28. Okuda H, Nakanishi T, Takatsu K, et al. Comparison of clinicopathological features of patients with hepatocellular carcinoma seropositive for alpha-fetoprotein alone and those seropositive for desgamma-carboxy prothrombin alone. J Gastroenterol Hepatol. 2001;16:1290-6.

29. Evdokimova VN, Liu Y, Potter DM, Butterfield LH. AFP-specific CD4+ helper T-cell responses in healthy donors and HCC patients. J Immunother. 2007;30:425-37.

30. Shim SJ, Seong J, Han KH, Chon CY, Suh CO, Lee JT. Local radiotherapy as a complement to incomplete transcatheter arterial chemoembolization in locally advanced hepatocellular carcinoma. Liver Int. 2005;25:1189-96.

31. Kawaguchi T, Ohkawa K, Imanaka K, et al. Lipiodol accumulation and transarterial chemoembolization efficacy for HCC patients. Hepatogastroenterology. 2012;59:219-23.

32. Kim BK, Kim SU, Kim KA, et al. Complete response at first chemoembolization is still the most robust predictor for favorable outcome in hepatocellular carcinoma. J Hepatol. 2015;62:1304-10.

\section{Tables}

Table 1 Baseline characteristics of HCC patients treated by TACE with CBCT assistance (CBCT-TACE) and patients treated by TACE with DSA alone (DSA-TACE) 


\begin{tabular}{|c|c|c|c|}
\hline \multirow[t]{2}{*}{ Prognostic factors } & СВСТ-TACE & DSA-TACE & \multirow[t]{2}{*}{$p$ value } \\
\hline & $n=196$ & $n=141$ & \\
\hline Age, years, mean (range) & $62.3(21-91)$ & $63.3(37-90)$ & 0.407 \\
\hline Gender & & & 0.414 \\
\hline Male & $140(71)$ & $94(67)$ & \\
\hline Female & $56(29)$ & $47(33)$ & \\
\hline Alcohol drinking & & & 0.978 \\
\hline No & $164(84)$ & $119(84)$ & \\
\hline Yes & $32(16)$ & $22(16)$ & \\
\hline Hepatitis B virus carrier & & & 0.614 \\
\hline No & $99(51)$ & $76(54)$ & \\
\hline Yes & $97(49)$ & $65(46)$ & \\
\hline Hepatitis C virus carrier & & & 0.773 \\
\hline No & $142(72)$ & $105(75)$ & \\
\hline Yes & $54(28)$ & $36(25)$ & \\
\hline Child-Pugh Class & & & 0.283 \\
\hline$A(5-6)$ & $144(73)$ & $96(68)$ & \\
\hline B (7) & $37(19)$ & $27(19)$ & \\
\hline$B(8-9)$ & $15(8)$ & $18(13)$ & \\
\hline ALBI score & & & 0.780 \\
\hline 1 & $53(27)$ & $35(25)$ & \\
\hline 2 & $131(67)$ & $95(67)$ & \\
\hline 3 & $12(6)$ & $11(8)$ & \\
\hline Total bilirubin, (mg/dl), median (range) & $0.87(0.18-2.87)$ & $0.98(0.20-2.95)$ & 0.154 \\
\hline Albumin, (ng/ml), median (range) & $3.6(2.2-4.7)$ & $3.4(1.8-4.8)$ & 0.064 \\
\hline INR, median (range) & $1.25(1.0-2.2)$ & $1.22(0.9-2.0)$ & 0.052 \\
\hline Platelet count, $\times 10^{3} / \mathrm{mm}^{3}$, median (range) & $99(30-435)$ & $106(35-318)$ & 0.853 \\
\hline Serum AFP level (ng/ml) & & & 0.241 \\
\hline$<20$ & $96(49)$ & $56(40)$ & \\
\hline $20-200$ & $\begin{array}{l}57(29) \\
\text { ge } 16 / 21\end{array}$ & $48(34)$ & \\
\hline
\end{tabular}




\begin{tabular}{llll}
$>$ 200 & $43(22)$ & $37(26)$ & 0.222 \\
\hline Ascites & & & \\
No & $173(88)$ & $117(83)$ & \\
Yes & $23(12)$ & $24(17)$ & \\
\hline Size of largest tumor, mm, median (range) & $3.1(1.1-7.0)$ & $3.2(1.1-7.0)$ & 0.157 \\
\hline Tumor number & & & 0.179 \\
1 & $108(55)$ & $72(51)$ & \\
2-3 & $64(33)$ & $58(41)$ & 1.000 \\
$4-5$ & $24(12)$ & $11(8)$ & \\
\hline Site of tumors & & & \\
Unilobar & $165(84)$ & $118(84)$ & 0.547 \\
Bilobar & $31(16)$ & $23(16)$ & \\
\hline Up-to-7 criteria & & $109(77)$ & \\
Within & $158(81)$ & $32(23)$ & \\
Beyond & $38(19)$ & $71(50)$ & \\
\hline BCLC-staging & & $70(50)$ & \\
Stage A & $115(59)$ & & \\
Stage B & $81(41)$ & & \\
\hline & & & \\
\hline
\end{tabular}

Data are presented as number (\%) unless otherwise indicated.

$A L B I$ albumin-bilirubin, INR international normalized ratio, $A F P$ alpha-fetoprotein, $B C L C$ Barcelona Clinic Liver Cancer

* Likelihood ratio test

Table 2 Procedural details and tumor responses of the HCC patients treated by TACE with CBCT assistance (CBCT-TACE) and patients treated by TACE with DSA alone (DSA-TACE) 


\begin{tabular}{|c|c|c|c|}
\hline & $\begin{array}{l}\text { СВCT-TACE } \\
(\mathrm{n}=196)\end{array}$ & $\begin{array}{l}\text { DSA-TACE } \\
(n=141)\end{array}$ & $p$ value \\
\hline Lipiodol, ml, mean (range) & $7.5 \pm 3.4(2-16)$ & $7.8 \pm 3.3(2-16)$ & 0.068 \\
\hline Doxorubicin dose, mg, mean (range) & $21.6 \pm 10.6(5-50)$ & $33.6 \pm 17.1(5-50)$ & $<0.001^{*}$ \\
\hline Number of treated hepatic segments & & & 0.150 \\
\hline 1 & $107(54)$ & $76(54)$ & \\
\hline 2 & $68(35)$ & $59(42)$ & \\
\hline 3 & $16(8)$ & $5(3)$ & \\
\hline 4 & $5(3)$ & $1(1)$ & \\
\hline Number of cine acquisitions, mean (range) & $4.6(1-14)$ & $5.1(2-17)$ & $0.017 *$ \\
\hline Number of CBCT acquisitions, mean (range) & $3.4(1-11)$ & & \\
\hline Fluoroscopic time, mins, mean (range) & $16.3(4.1-60.4)$ & $12.4(3.3-54.4)$ & $<0.001^{*}$ \\
\hline Post embolization syndrome (\%) & & & 0.920 \\
\hline Yes & $19(10)$ & $15(11)$ & \\
\hline No & $177(90)$ & $126(89)$ & \\
\hline Tumor response ${ }^{a}$ & & & $<0.001 *$ \\
\hline $\mathrm{CR}$ & $131(67)$ & $31(22)$ & \\
\hline PR & $55(28)$ & $67(48)$ & \\
\hline $\mathrm{SD}$ & $7(4)$ & $13(9)$ & \\
\hline PD & $3(1)$ & $30(21)$ & \\
\hline Additional TACE, times, mean (range) & $2.7(1-10)$ & $3.0(1-14)$ & 0.400 \\
\hline
\end{tabular}

Data are presented as number (\%) unless otherwise indicated.

$C R$ complete response, $\mathrm{PR}$ partial response, $S D$ stable disease, $P D$ progressive disease, CBCT-TACE conebeam computed tomography assisted transarterial chemoembolization, DSA-TACE digital subtraction angiography assisted transarterial chemoembolization

a Tumor response defined by modified Response Evaluation Criteria in Solid Tumors Criteria (mRECIST).

Table 3. Results of univariate and multivariate analysis on potential prognostic factors affecting survival after chemoembolization 
Prognostic factors

\begin{tabular}{|c|c|c|c|c|c|}
\hline & \multirow[b]{2}{*}{ of cases } & & & & \\
\hline & & $\mathrm{HR}(95 \% \mathrm{Cl})$ & $\begin{array}{l}p \\
\text { value }\end{array}$ & $\mathrm{HR}(95 \% \mathrm{Cl})$ & $\begin{array}{l}p \\
\text { value }\end{array}$ \\
\hline Age $(\leq 65 />65$ years $)$ & $200 / 137$ & $\begin{array}{l}0.91(0.70- \\
1.20)\end{array}$ & 0.512 & & \\
\hline Gender (male/female) & $234 / 103$ & $\begin{array}{l}0.88(0.66- \\
1.18)\end{array}$ & 0.392 & & \\
\hline Alcohol drinking (no/yes) & $283 / 54$ & $\begin{array}{l}0.88(0.61- \\
1.25)\end{array}$ & 0.470 & & \\
\hline Hepatitis B virus carrier (no/yes) & $175 / 162$ & $\begin{array}{l}0.99(0.76- \\
1.29)\end{array}$ & 0.956 & & \\
\hline Hepatitis C virus carrier (no/yes) & $247 / 90$ & $\begin{array}{l}1.01(0.75- \\
1.35)\end{array}$ & 0.963 & & \\
\hline Child-Pugh score (A/B) & $240 / 97$ & $\begin{array}{l}0.56(0.43- \\
0.74)\end{array}$ & $<0.001$ & & \\
\hline ALBI score $(1,2$, and 3$)$ & $88 / 249$ & $\begin{array}{l}0.49(0.35- \\
0.69)\end{array}$ & $<0.001$ & $\begin{array}{l}0.48(0.34- \\
0.68)\end{array}$ & $\hat{0}_{0.001 *}$ \\
\hline $\begin{array}{l}\text { Total Bilirubin }(\leq 1.0 /> \\
1.0 \mathrm{mg} / \mathrm{dl})\end{array}$ & $194 / 143$ & $\begin{array}{l}0.59(0.45- \\
0.77)\end{array}$ & $<0.001$ & & \\
\hline Albumin $(>3.5 / \leq 3.5 \mathrm{~g} / \mathrm{dl})$ & $159 / 178$ & $\begin{array}{l}0.47(0.36- \\
0.62)\end{array}$ & $<0.001$ & & \\
\hline $\operatorname{INR}(\leq 1.2 />1.2)$ & 138/199 & $\begin{array}{l}0.61(0.46- \\
0.80)\end{array}$ & $\begin{array}{l}< \\
0.001\end{array}$ & & \\
\hline Platelet $\left(>10^{5} / \leq 10^{5} \mathrm{~mm}^{3}\right)$ & $170 / 167$ & $\begin{array}{l}0.81(0.62- \\
1.05)\end{array}$ & 0.113 & & \\
\hline $\begin{array}{l}\text { Serum AFP level }(\leq 200 />200 \\
\mathrm{ng} / \mathrm{ml})\end{array}$ & $257 / 80$ & $\begin{array}{l}0.73(0.54- \\
0.99)\end{array}$ & 0.041 & $\begin{array}{l}0.63(0.46- \\
0.86)\end{array}$ & $0.003^{*}$ \\
\hline Ascites (no/yes) & $290 / 47$ & $\begin{array}{l}0.60(0.42- \\
0.86)\end{array}$ & 0.005 & & \\
\hline BCLC-staging (0-A/B) & $186 / 151$ & $\begin{array}{l}0.64(0.49- \\
0.83)\end{array}$ & $<.001$ & & \\
\hline Size of largest tumor $(\leq 3 />3 \mathrm{~cm})$ & $151 / 186$ & $\begin{array}{l}0.71(0.54- \\
0.93)\end{array}$ & 0.012 & & \\
\hline Single tumor (yes/no) & $180 / 157$ & $\begin{array}{l}0.71(0.54- \\
0.92)\end{array}$ & 0.011 & & \\
\hline Unilobar involvement (yes/no) & $283 / 54$ & $\begin{array}{l}0.82(0.58- \\
1.16)\end{array}$ & 0.262 & & \\
\hline Up-to-7 criteria (within/beyond) & $267 / 70$ & $\begin{array}{l}0.88(0.64- \\
1.22)\end{array}$ & 0.447 & & \\
\hline
\end{tabular}


CR at 1 month after TACE (yes/no)

CBCT assisted TACE (yes/no)
$162 / 175$

$0.34(0.26-$
$0.46)$

$196 / 141$
$0.34(0.26-$ $0.44)$
$<$

0.001

$0.55(0.40-$
$0.75)$

$<$

$0.001^{*}$

$\begin{array}{lll}< & 0.38(0.28- & < \\ 0.001 & 0.51) & 0.001 *\end{array}$

$H R$ hazard ratio, $A L B /$ albumin-bilirubin, INR international normalized ratio, $A F P$ alpha-fetoprotein, $B C L C$ Barcelona Clinic Liver Cancer, CR complete response, CBCT cone-beam computed tomography, TACE transarterial chemoembolization, 95\% C/95\% confidence interval

${ }^{*}$ Likelihood ratio test

Figures
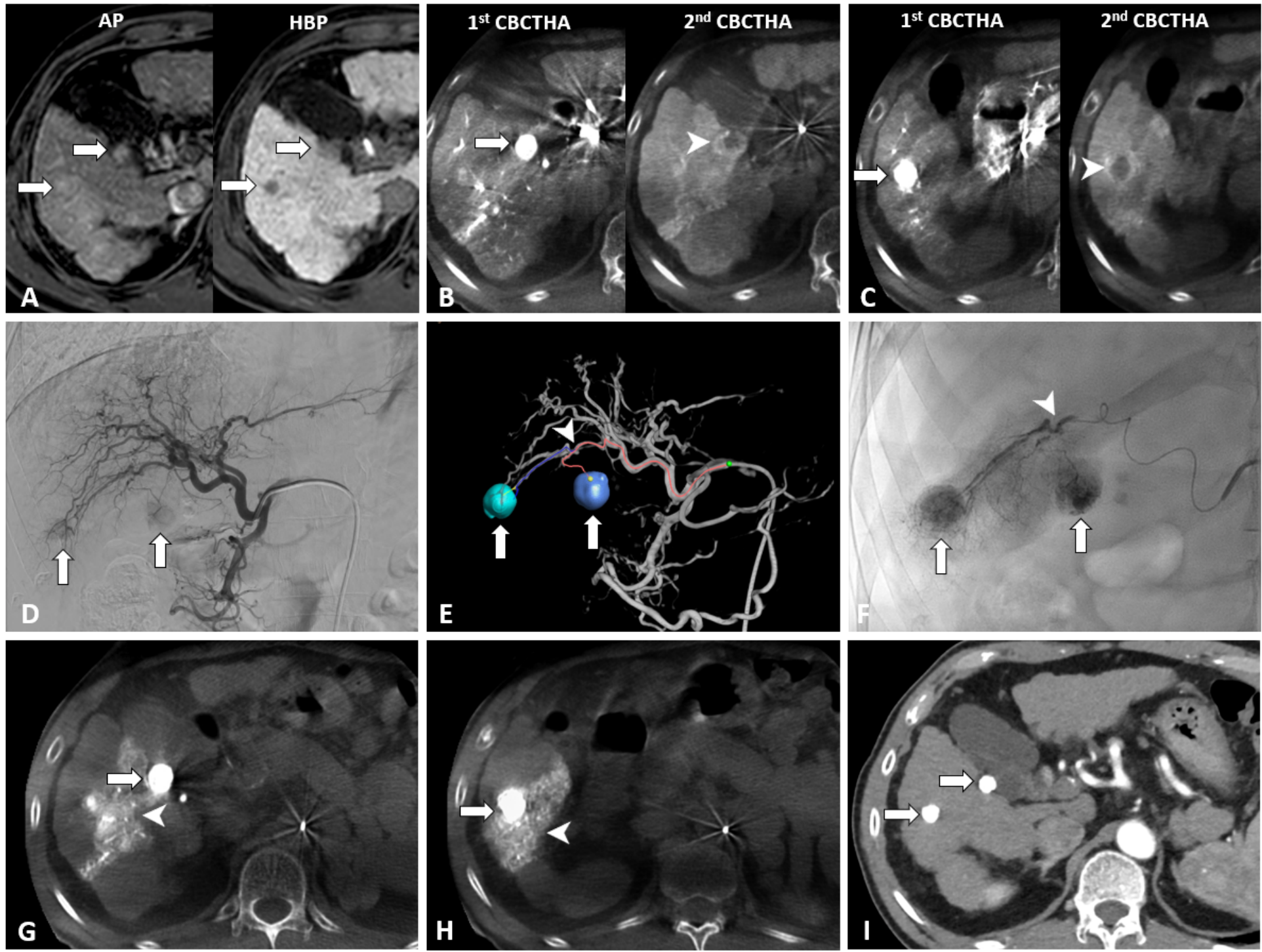

Figure 1 
Cone-beam computed tomography (CBCT) with automated tumor-feeder detection software assisted transarterial chemoembolization (TACE). (A) Gadoxetic acid-enhanced MRI in the arterial phase in a 58year-old male with chronic viral hepatitis-B cirrhosis showing two hyperenhancing nodules (arrows) in liver segment 5 . Hepatobiliary phase imaging at 20 minutes after injection shows two hypointense nodules (arrows) against the background of enhancing liver parenchyma, which may indicate HCC. (B-C) Dual-phase CBCT during hepatic arteriography could depict all tumors (arrows) with corona enhancement (arrowheads). (D) Common hepatic arteriogram showed two faint tumor stains (arrows). However, the tumor-feeders were unclear. (E) Automated tumor-feeder detection software identified the feeders (arrowhead) of each tumor (arrows). (F) Tumor-feeder (arrowhead) was selectively embolized during TACE. Complete tumor staining (arrows) was demonstrated from spot image on digital subtraction angiography. $(\mathrm{G}-\mathrm{H}) \mathrm{CBCT}$ immediately after TACE showed dense iodized oil accumulation in all tumors (arrows) with a sufficient safety margin (arrowheads). (I) Enhanced CT performed 1 year after selective TACE showed dense iodized oil accumulation in all tumors without tumor recurrence

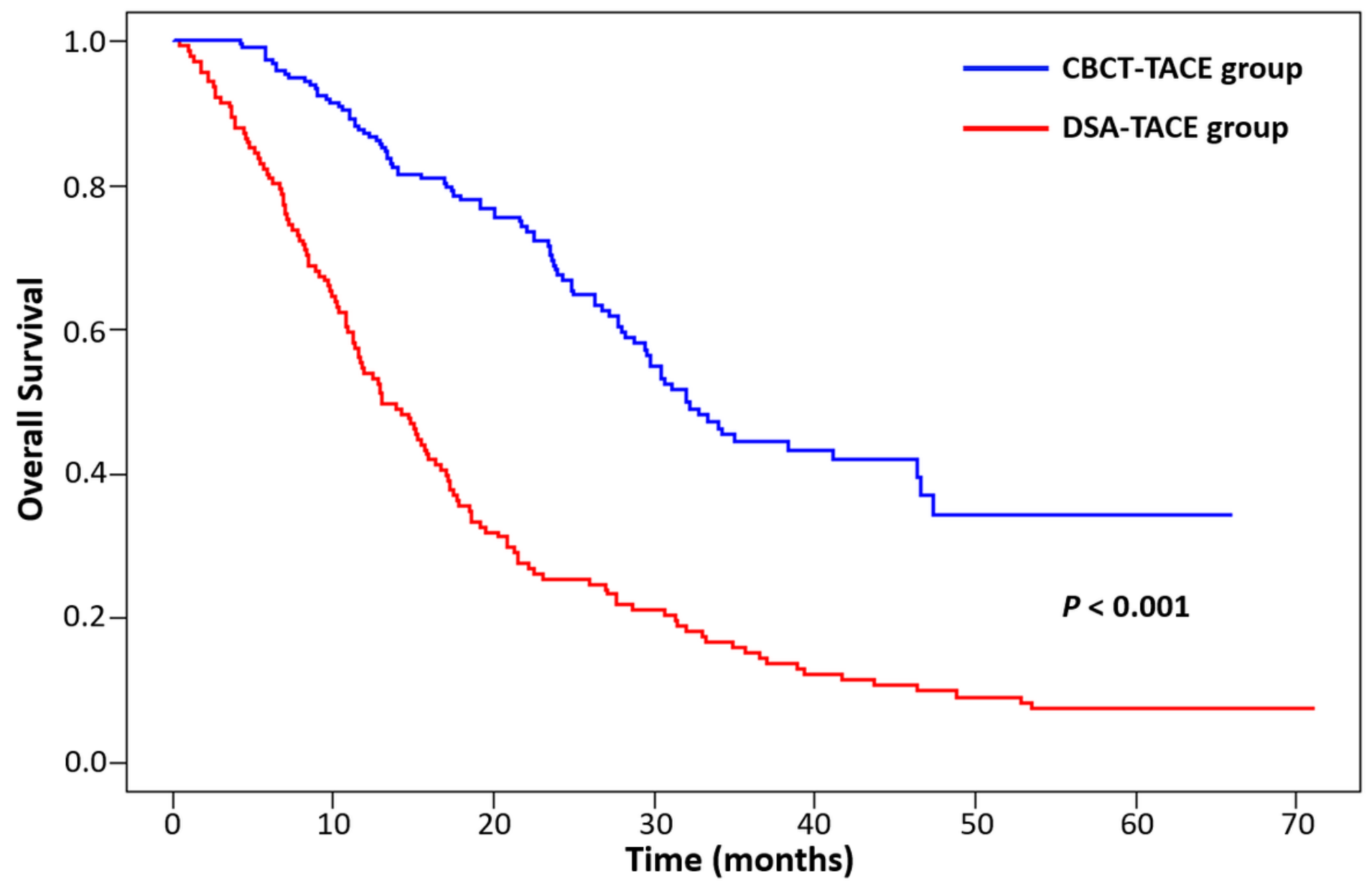

Figure 2

Cumulative overall survival of HCC treated with selective transarterial chemoembolization (TACE) with cone-beam computed tomography (CBCT) and automated tumor-feeder detection software assistance (CBCT-TACE group) or with digital subtraction angiography (DSA-TACE group) 